\title{
Coherent oscillation by self-induced gratings in the photorefractive crystal $\mathrm{BaTiO}_{3}$
}

\author{
Jeffrey O. White, Mark Cronin-Golomb, Baruch Fischer, and Amnon Yariv \\ Department of Applied Physics, California Institute of Technology, Pasadena, California 91125
}

(Received 10 August 1981; accepted for publication 14 December 1981)

\begin{abstract}
We report here the demonstration of several new optical oscillator configurations including a unidirectional ring oscillator and a self-pumped phase conjugate mirror. The gain medium is $\mathrm{BaTiO}_{3}$, pumped by a $632.8-\mathrm{nm} \mathrm{He}-\mathrm{Ne}$ laser at power levels down to $50 \mu \mathrm{W}$.
\end{abstract}

PACS numbers: 42.65. - k, 42.55.Rz, 42.60.Da, 42.60.By

The possibility of phase conjugate mirrors (PCM's) with reflectivities exceeding unity was predicted by Yariv and Pepper, ${ }^{1}$ and subsequently observed by Bloom, Liao, and Economou, ${ }^{2}$ and by Pepper, Fekete, and Yariv. ${ }^{3}$ The properties of resonators with PCM's have been studied experimentally and theoretically. ${ }^{4}$ Early work employed high intensity $Q$ switched lasers as the pump beams. ${ }^{5}$ Because of formal similarities of four-wave mixing in third-order optically nonlinear media, and in real-time holographic media, ${ }^{6}$ the considerations of these early works may be carried over to the analysis at PCM's based on real-time holography in photorefractive media. For example, in a recent experiment with $\mathrm{BaTiO}_{3}$ as the holographic photorefractive medium, Feinberg and Hellwarth ${ }^{7}$ demonstrated a PCM with reflectivity exceeding unity using a $\mathrm{cw}$ argon laser at $514.5 \mathrm{~nm}$ for the pump beams. They used this mirror in conjunction with an ordinary mirror to demonstrate a cw oscillator of the type shown in Fig. 1(a) and to examine its behavior with intracavity spatial filters and phase distorters.

In this letter, we report the demonstration of several new oscillator configurations involving $\mathrm{BaTiO}_{3}$ : (i) an oscillator using two conventional mirrors in which the gain is provided by two pumping beams [Fig. 1(b)]; (ii) an oscillator pumped by a single beam using two conventional mirrors [Fig. 1(c)]; (iii) a unidirectional ring oscillator pumped by a single beam [Fig. 1(d)]; (iv) a ring oscillator pumped with two counter-propagating beams [Fig. 1(e)].

We pumped these oscillators, as well as the one shown in Fig. 1(a) with a low-power $(\sim 1 \mathrm{~mW}) \mathrm{He}-\mathrm{Ne}$ laser operating at $632.8 \mathrm{~nm}$. This is the first demonstration of $\mathrm{cw} \mathrm{BaTiO}_{3}$ oscillators with the $\mathrm{He}-\mathrm{Ne}$ laser. This new oscillator technology has thus been brought within the reach of even modestly equipped laboratories.

The above oscillators do not operate by the simulated emission of radiation. Small signals experience gain when one or both pumps are diverted in the proper direction by Bragg reflection from self-induced index gratings as explained below.

In the above oscillators, the amplified signal is fed back by one or more mirrors in the form of a resonator. Our resonators were constructed with plane mirrors so that they were unstable to diffraction loss, except for any stabilization due to the phase-conjugating properties of the crystal. Experiments are currently in progress to examine the effect of resonator stabilization using spherical mirrors. We tried one resonator [Fig. 1(f)] in which the feedback was via a Bragg reflection inside the crystal, as in DFB lasers. No oscillation has been observed yet.

The crystal of $\mathrm{BaTiO}_{3}$ measured $7 \times 4.5 \times 4 \mathrm{~mm} .{ }^{8}$ It was poled into a single domain so that the $c$ axis was parallel to the $4 \mathrm{~mm}$ side. In order to make use of the large electro-optic coefficient $r_{42}=820 \times 10^{-12} \mathrm{mV}^{-1},{ }^{9}$ the crystal was oriented so that the wave vectors of the holographic gratings were not parallel to any crystal axis. The polarization of all beams was extraordinary, and they were made to travel predominantly in the direction of the $c$ axis. The polarity of the $c$ axis determines the direction in which power is transferred in two beam coupling experiments. ${ }^{10}$ The four beams shown in Fig. 2 enter and leave the crystal through the two parallel crystal faces which measure $7 \times 4.5 \mathrm{~mm}$. Coupling is also possible when the beams 1 and 4 enter different faces, although the interaction region may be reduced.

In each resonator pump $A_{1}$ is delivered directly from the laser and has a diameter of approximately $3 \mathrm{~mm}$. Table I shows the steady-state intensities of the oscillation beams $A_{3}$ and $A_{4}$ as they left the cuvette, and the time taken to reach steady state. They were measured either directly or through $1 \%$ transmitting mirrors. In each case, the angle $\alpha$ was $168^{\circ}$ and $\beta$ was $160^{\circ}$.
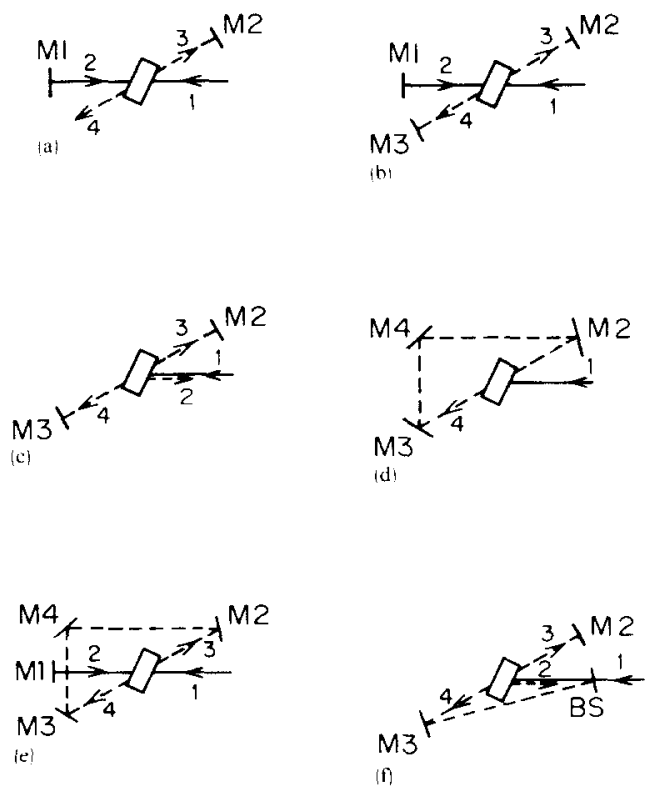

FIG. 1. Arrangement of barium titanate crystal (rectangle) mirrors (labelled as referred to in text) and light beams of the oscillators (a)-(f) as described in the text. 


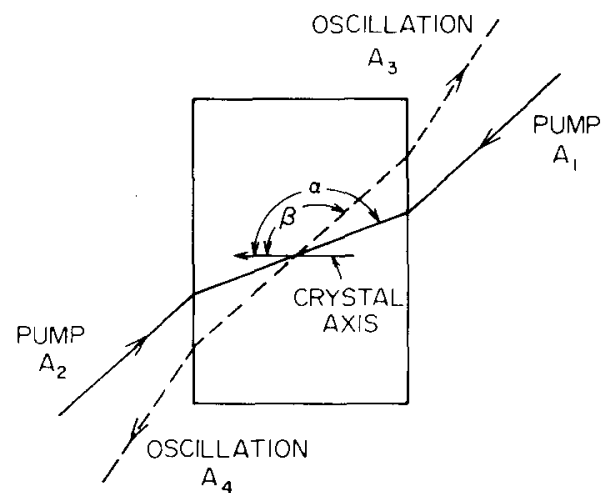

FIG. 2. Expanded view of the interaction region common to each of the oscillators, showing the pumping beams (solid), oscillation beams (dashed), and the relative orientation of the $c$ axis of the crystal.

In the resonator of Fig. 1(a) pump $A_{2}$ is provided by retroreflecting $A_{1}$ at the mirror $M 1$. It is known that in this configuration, beam $A_{4}$ can experience amplified-phase conjugate reflection. ${ }^{6}$ Oscillation can thus build up between $M 2$ and the crystal, provided the reflection gain is sufficient to compensate for the losses in the oscillator. When the mirror $M 2$ is placed on the opposite side of the crystal $\left(\beta \rightarrow \beta+180^{\circ}\right)$, the oscillation strength increases by a factor of 10 . A preliminary check indicated that oscillation was possible in the angular ranges $155^{\circ}<\beta<160^{\circ}$ and $-22^{\circ}<\beta<-12^{\circ}$. In terms of angles measured outside the crystal, the oscillation could be observed over a total range of $80^{\circ}$. We note here that the threshold behavior of this device is quite different from that of lasers. It was observed that oscillation builds up even with the very low pumping power of 50 $\mu \mathrm{W}$ in $\mathrm{A}_{1}$, although the time taken to reach steady state in that case was as long as eight minutes.

Since one mirror on either side of the crystal is sufficient to obtain oscillation, it was not surprising to observe oscillation in the configuration of Fig. 1(b). It was surprising, however, to see oscillation build up even when the counterpropagating pump $A_{2}$ is removed as in Fig. 1(c). We believe that the mechanism of oscillation in this case is as follows: Beam $A_{4}$ interacts in the crystal with the pump $A_{1}$ to form a photorefractive grating. The direction of the $c$ axis is such that, as explained by two-beam coupling theory, ${ }^{10}$ beam $A_{4}$ is amplified by $A_{1}$. This amplified beam is fed back into the direction of $A_{4}$ by mirrors $M 3$ and $M 2$. If the gain exceeds the absorption and reflection losses, an oscillation will build up.

It was observed that a fourth beam, $A_{2}$, was generated, traveling backward relative to $A_{1}$, and having up to $10 \%$ of the intensity of $A_{1}$. This is presumably light scattered from $A_{3}$ by the grating formed between $\mathrm{A}_{1}$ and $A_{4}$. This device can be viewed as a signal-powered phase conjugate mirror, i.e., a "black box" which reflects a phase conjugate of an input

TABLE I. Oscillation strengths and rise times for the resonators of Fig. 1.

\begin{tabular}{llllll}
\hline \hline & (a) & (b) & (c) & (d) & (e) \\
\hline$I_{3}(\mathrm{~mW})$ & 0.1 & 0.8 & 0.3 & 0 & 0.2 \\
$I_{4}(\mathrm{~mW})$ & 2 & 3 & 3 & 3 & 3 \\
$7(\mathrm{sec})$ & 8 & 8 & 100 & 8 & 8 \\
\hline \hline
\end{tabular}

wave without a coherent pump source. It is important to note that the orientation of the parallel mirror/crystal combination with respect to beam $A_{1}$ is not critical. The oscillator acts as a PCM with a wide spatial bandwidth; we have observed that two beams incident simultaneously on this PCM were reflected with $10 \%$ reflectivity, even though they were separated in angle by about ten degrees.

In Fig. 1(d) we show a unidirectional ring oscillator. Its operation is due to the preferential amplification by $A_{1}$ of $A_{4}$ compared to that of $A_{3}$. When two pumps are provided, Fig. $1(\mathrm{e})$, oscillation is observed in both directions.

The unidirectional ring oscillator can be described by two-beam coupling theory. ${ }^{10}$ Consider coupling of $A_{1}$ and $A_{4}$. Let $\theta_{1}$ be the angle between beam $A_{1}$ and the normal to the crystal surface. Let $\theta_{4}$ be the corresponding angle for $A_{4}$. Let $z$ be the distance measured along the normal to the crystal surface, and let the length of the crystal along this direction be $l$. We write the total optical field as the sum of two beams

$$
\begin{aligned}
E(r, t)= & A_{1}(\mathbf{r}) e^{i\left(\mathbf{k}_{\mathbf{k}_{1}} \mathbf{r}-\omega t\right)}+\text { c.c. } \\
& +A_{4}(\mathbf{r}) e^{i\left(\mathbf{k}_{4} \cdot \mathbf{r}-\omega t\right)}+\text { c.c. }
\end{aligned}
$$

The interference of these beams in a photorefractive crystal creates a phase grating which may be represented by a perturbation on the mean refractive index.

$$
\Delta n=\left(b e^{i \phi} / I_{0}\right) A_{1}^{*} A_{4} e^{i k \cdot r}+\text { c.c., }
$$

where $\mathbf{k}$, the wave vector of the grating, is equal to $\mathbf{k}_{4}-\mathbf{k}_{1}$. The phase $\phi$ indicates the degree to which the grating is shifted spatially with respect to the light interference pattern. In photorefractive media which can operate by diffusion only, e.g., $\mathrm{BaTiO}_{3}$, the magnitude of $\phi$ is $\pi / 2$ with its sign depending on the direction of the $c$ axis. $I_{0}$ is the intensity sum $\left|A_{1}\right|^{2}+\left|A_{4}\right|^{2}$. The parameter $b$ depends on the magnitude of the grating vector and its direction and on the material properties of the crystal, e.g., the electro-optic coefficient. In Ba$\mathrm{TiO}_{3}$, holographic two-beam coupling was first observed in $1980 .{ }^{11}$ In that previous study the $k$ vector of the holographic grating was arranged to be parallel to the $c$ axis of the crystal, so that no advantage was taken at the large electrooptic coefficient $r_{42}$.

Using the standard slowly varying field approximation and the scalar wave equation, we can obtain the following coupled wave equations for $A_{1}$ and $A_{4}{ }^{6,10}$

$$
\begin{aligned}
& \frac{d A_{1}}{d z}=-\frac{i b \omega e^{i \phi}}{2 c I_{0} \cos \theta} A_{1}\left|A_{4}\right|^{2}, \\
& \frac{d A_{4}}{d z}=-\frac{i b \omega e^{-i \phi}}{2 c I_{0} \cos \theta} A_{4}\left|A_{1}\right|^{2},
\end{aligned}
$$

where we have taken $\theta_{1}=\theta_{4}=\theta$ for simplicity. From (3) we can derive equations for the intensities $I_{1}=\left|A_{1}\right|^{2}$ and $I_{4}=\left|A_{4}\right|^{2}$.

$$
\begin{aligned}
& \frac{d I_{1}}{d z}=-\frac{\Gamma I_{1} I_{4}}{I_{0}}, \\
& \frac{d I_{4}}{d z}=\frac{\Gamma I_{1} I_{4}}{I_{0}},
\end{aligned}
$$


where $\Gamma=-b \omega \sin \phi / c \cos \theta$, so that $I_{4}$ exhibits gain for positive $\Gamma$. We note that the total energy is conserved,

$$
\frac{d I_{0}}{d z}=0,
$$

so that $I_{0}$ is constant. The solution of Eqs. (3) and (4) is

$$
\begin{aligned}
& I_{1}(z)=\frac{I_{0}}{1+\left[I_{4}(0) / I_{1}(0)\right] e^{\Gamma_{z}}}, \\
& I_{4}(z)=\frac{I_{0}}{1+\left[I_{1}(0) / I_{4}(0)\right] e^{-\Gamma_{z}}} .
\end{aligned}
$$

When we apply boundary condition appropriate to a ring oscillator

$$
I_{4}(0)=(1-L) I_{4}(l),
$$

where $L$ represents the cavity's fractional linear intensity loss per round trip we find that the ratio of oscillating energy to pumping energy is

$$
\frac{I_{4}(0)}{I_{1}(0)}=\frac{1-e^{-\Gamma t}}{L}-1 .
$$

We note that $I_{4}(0) / I_{1}(0)$ is infinite for $L=0$ and becomes zero, i.e., no oscillation at $L=1-e^{-\Gamma l}$.

In conclusion, we have demonstrated a number of new oscillators involving four-wave mixing in $\mathrm{BaTiO}_{3}$. Oscilla- tion is achieved with a low-power He-Ne laser at $632.8 \mathrm{~nm}$ supplying the pump beam(s).

This work was supported by the Air Force Office of Scientific Research and the Army Research Office. B. Fischer would like to acknowledge the support of the Weizmann post-doctoral fellowship and M. Cronin-Golomb the support of the University of Sydney. This paper is dedicated to Alice and Esther.

\footnotetext{
'A. Yariv and D. M. Pepper, Opt. Lett. 1, 16 (1977).

${ }^{2}$ D. M. Bloom, P. F. Liao, and N. P. Economou, Opt. Lett. 2, 58 (1978).

${ }^{3}$ D. M. Pepper, D. Fekete, and A. Yariv, Appl. Phys. Lett. 33, 41 (1978).

${ }^{4}$ P. A. Bélanger, A. Hardy, and A. E. Siegman, Appl. Opt. 19, 602 (1980).

${ }^{5} \mathrm{~J}$. AuYeung, D. Fekete, D. M. Pepper, and A. Yariv, IEEE J. Quantum

Electron. QE-15, $1180(1979)$.

${ }^{6} B$. Fischer, M. Cronin-Golomb, J. O. White, and A. Yariv, Opt. Lett. 6, $519(1981)$.

${ }^{7}$ J. Feinberg and R. W. Hellwarth, Opt. Lett. 5, 319 (1980).

${ }^{8}$ Sanders Associates, 95 Canal Street, Nashua, NH 03060.

${ }^{9} A$. Yariv, Quantum Electronics, 2nd ed. (Wiley, New York, 1975).

${ }^{10}$ V. L. Vinetskii, N. V. Kukhtarev, S. G. Odulov, and M. S. Soskin, Usp.

Fiz. Nauk 129, 113 (1979) [Sov. Phys. Usp. 22, 742 (1979)]

"J. Feinberg, D. Heiman, A. R. Tanguay, Jr., and R. W. Hellwarth, J. Appl. Phys. 51, 1297 (1980).
}

\section{Effect of superluminescence on the modulation response of semiconductor lasers}

Kam Y. Lau

Ortel Corporation, Alhambra, California 91803

Amnon Yariv

California Institute of Technology, Pasadena, California 91125

(Received 6 November 1981; accepted for publication 5 January 1982)

The small-signal modulation response of semiconductor lasers with a very small mirror reflectivity is analyzed. Superluminescent effects inside the laser cavity provide yet another mechanism for damping relaxation oscillation resonance. These results can serve as useful guides in designing high frequency semiconductor lasers.

PACS numbers: 42.55.Px, 42.60.Fc, 42.80.Sa

Semiconductor lasers are potentially important as light source for optical communication systems. Consequently, methods for improving the modulation response of these devices are topics of great interest. The small-signal modulation behavior of the laser is well established experimentally and theoretically. ${ }^{\prime}$ The absence of a resonance peak in the modulation response of some lasers has been attributed to a variety of causes including the high spontaneous emission factor and/or lateral carrier diffusion. In this letter we point out yet another mechanism that can lead to a flat frequency response. This mechanism involves the nonuniform distribution of photons and carriers along the length of a laser cavity with a small mirror reflectivity. This effect is particularly important when one attempts to increase the modulation speed by reducing the end-facet reflectivities in order to reduce the lifetime of the cavity. ${ }^{2}$

Previous considerations of nonuniform distribution of photons and carriers occurred in the context of superluminescent light emitting diodes (LED's)-diodes with totally suppressed mirror feedback to prevent lasing. ${ }^{3-7}$ Total suppression of mirror feedback is unattainable in practice. A very low reflectivity at one mirror can be obtained by leaving a long section of the laser unpumped ${ }^{3}$; nevertheless, scattering from material defects and waveguide irregularities make 